\title{
Genetic variation in apolipoprotein A-I concentrations and risk of coronary artery disease
}

Minna K. Karjalainen ${ }^{1,2,3, *}$, Ph.D., Michael V. Holmes ${ }^{4,5,6,7, *,+}$, M.D., Ph.D., Qin Wang ${ }^{1,2,3,8}$, Ph.D., Olga Anufrieva ${ }^{1,2,3}$, M.Sc, Mika Kähönen ${ }^{9}$, M.D., Ph.D., Terho Lehtimäki ${ }^{10}$, M.D., Ph.D.,

Aki S. Havulinna ${ }^{11,12}$, D.Tech., Kati Kristiansson ${ }^{11}$, Ph.D., Veikko Salomaa ${ }^{11}$, M.D., Ph.D., Markus Perola ${ }^{11,13,14}$, M.D., Ph.D., Jorma S. Viikari ${ }^{15,16}$, M.D., Ph.D., Olli T. Raitakari ${ }^{17,18}$, M.D., Ph.D., Marjo-Riitta Järvelin²,3,19,20,21, M.D., Ph.D., Mika Ala-Korpela1,2,3,7,8,22,23,24,\#,+, Ph.D., Johannes Kettunen ${ }^{1,2,3,11, \#,+}$, Ph.D.

* joint first authors; \# joint senior authors; + corresponding authors

1. Computational Medicine, Faculty of Medicine, University of Oulu, Oulu, Finland

2. Center for Life Course Health Research, Faculty of Medicine, University of Oulu, Oulu, Finland

3. Biocenter Oulu, University of Oulu, Oulu, Finland

4. Medical Research Council Population Health Research Unit, University of Oxford, Oxford, UK

5. Clinical Trial Service Unit \& Epidemiological Studies Unit, Nuffield Department of Population Health, University of Oxford, Oxford, UK

6. National Institute for Health Research, Oxford Biomedical Research Centre, Oxford University Hospital, Oxford, UK

7. Medical Research Council Integrative Epidemiology Unit at the University of Bristol, Bristol, UK

8. Systems Epidemiology, Baker Heart and Diabetes Institute, Melbourne, VIC, Australia

9. Department of Clinical Physiology, Tampere University Hospital, and Finnish Cardiovascular Research Center Tampere, Faculty of Medicine and Health Technology, Tampere University, Tampere, Finland

10. Department of Clinical Chemistry, Fimlab Laboratories, and Finnish Cardiovascular Research Center Tampere, Faculty of Medicine and Health Technology, Tampere University, Tampere, Finland 
11. National Institute for Health and Welfare, Helsinki, Finland

12. Institute for Molecular Medicine Finland (FIMM-HiLIFE), Helsinki, Finland

13. Diabetes and Obesity Research Program, University of Helsinki, Helsinki, Finland

14. Estonian Genome Center, University of Tartu, Tartu, Estonia

15. Department of Medicine, University of Turku, Turku, Finland

16. Division of Medicine, Turku University Hospital, Turku, Finland

17. Research Centre of Applied and Preventive Cardiovascular Medicine, University of Turku, Turku, Finland

18. Department of Clinical Physiology and Nuclear Medicine, Turku University Hospital, Turku, Finland

19. Unit of Primary Health Care, Oulu University Hospital, OYS, Oulu, Finland

20. Department of Epidemiology and Biostatistics, MRC-PHE Centre for Environment and Health, School of Public Health, Imperial College London, London, UK

21. Department of Life Sciences, College of Health and Life Sciences, Brunel University London, UK

22. Population Health Science, Bristol Medical School, University of Bristol, Bristol, UK

23. NMR Metabolomics Laboratory, School of Pharmacy, University of Eastern Finland, Kuopio, Finland

24. Department of Epidemiology and Preventive Medicine, School of Public Health and Preventive Medicine, Faculty of Medicine, Nursing and Health Sciences, The Alfred Hospital, Monash University, Melbourne, VIC, Australia

SHORT TITLE: Apolipoprotein A-I and coronary artery disease

\author{
Abstract: 283 words \\ Manuscript word count: 6868 words \\ Figures: 4 \\ Tables: 0 \\ References: 48 \\ Online Supplement
}




\section{Correspondence:}

\section{Associate Professor Michael V. Holmes}

Medical Research Council Population Health Research Unit

University of Oxford

Oxford, UK

E-mail:michael.holmes@ndph.ox.ac.uk

Mobile: +44 7479531754

\section{Professor Mika Ala-Korpela}

Baker Heart and Diabetes Institute

Systems Epidemiology

75 Commercial Road

Melbourne, Victoria 3004

Australia

E-mail: mika.ala-korpela@baker.edu.au

Mobile: +61 452392966

\section{Professor Johannes Kettunen}

\section{Computational Medicine}

Center for Life Course Health Research

Faculty of Medicine

University of Oulu

Oulu, Finland

E-mail: johannes.kettunen@oulu.fi

Mobile: +358 505629718 


\section{ABSTRACT}

Rationale: Apolipoprotein A-I (apoA-I) infusions represent a potential novel therapeutic approach for the prevention of coronary artery disease (CAD) with phase III cardiovascular outcome trials currently underway. Although circulating apoA-I levels inversely associate with risk of CAD, the evidence base of this representing a causal relationship is lacking.

Objective: To assess the causal role of apoA-I in CAD using human genetics.

Methods and Results: We identified a variant (rs12225230) in APOA1 locus that associated with circulating apoA-I concentrations at GWAS significance $\left(P<5 \times 10^{-8}\right)$ in 20,370 Finnish participants and meta-analyzed our data with a previous genome-wide association study of apoA-I. We obtained genetic estimates of CAD from UK Biobank and CARDIoGRAMplusC4D (totaling 122,733 CAD cases) and conducted a two-sample Mendelian randomization analysis. We compared our genetic findings to observational associations of apoA-I with risk of $C A D$ in 918 incident $C A D$ cases among 11,535 individuals from population-based prospective cohorts. We also summarized the available evidence from randomized controlled trials (RCTs) of apoA-I infusion therapies reporting CAD events. ApoA-I was associated with a lower risk of CAD in observational analyses (HR 0.81; $95 \% \mathrm{Cl}: 0.75,0.88$; per 1-SD higher apoA-I), with the association showing a dose-response relationship. Rs12225230 associated with apoA-I concentrations (per-C allele beta 0.076 SD; SE: $0.013 ; P=1.5 \times 10^{-9}$ ) but not with potential confounders. In Mendelian randomization analyses, apoA-I was not related to risk of CAD (OR 1.13; 95\%Cl: 0.98, 1.30 per 1-SD higher apoA-I), which was different to the observational association (P-het<0.001). RCTs of apoA-I infusions did not show an effect on the risk of CAD. 
Conclusions: Genetic evidence fails to support a cardioprotective role for apoA-I. This casts doubt on the likely benefit of apoA-I infusion therapy in the ongoing phase III cardiovascular outcome trial.

KEY WORDS: Coronary artery disease, Apolipoprotein A-I, Mendelian randomization.

NON-STANDARD ABBREVIATIONS AND ACRONYMS: apoA-I, apolipoprotein A-I; CAD, coronary artery disease; eQTL, expression quantitative trait locus; MACE, major adverse cardiovascular event; MR, Mendelian randomization; NMR, nuclear magnetic resonance; pQTL, protein quantitative trait locus; SNP, single-nucleotide polymorphism. 


\section{INTRODUCTION}

Large-scale cardiovascular outcome trials ${ }^{1-4}$ and studies of human genetics ${ }^{5,6}$ do not support a causal role of high-density lipoprotein (HDL) cholesterol in coronary artery disease (CAD). However, it remains feasible that other aspects of $\mathrm{HDL}$, such as the functional attributes of HDL particles, might have atheroprotective effects. The ability of HDL particles to extract cholesterol from lipid-laden cells, so-called cholesterol efflux, has recently emerged as the most prominent new measure for HDL-related atheroprotectivity. ${ }^{7}$ Apolipoprotein A-I (apoAI) is a key functional apolipoprotein component of HDL particles and plays a central role in cholesterol efflux. ${ }^{8}$ This has led to anticipation that modification of circulating apoA-I might represent a novel therapeutic approach to the treatment and prevention of CAD. Notably, Pfizer reportedly paid over $1 \mathrm{bn}$ USD for the commercial rights for an apoA-I infusion technology. ${ }^{9}$

Recent results from phase II randomized controlled trials (RCTs) of apoA-I that used MDCO216 (recombinant apoA-I-Milano) and CER-001 (recombinant wild-type apoA-I) infusions suggest that the initial optimism may be misplaced. ${ }^{10,11}$ Trials of MDCO-216 and CER-001 failed to identify a beneficial effect of apoA-I infusion on the regression of coronary atherosclerosis as measured by intravascular ultrasonography, ${ }^{10,11}$ resulting in the termination of the development of these apoA-I products. A third apoA-I infusion therapy, CSL112, representing a modified form of native apoA-I from human plasma, is currently underway in a large phase III RCT (AEGIS-II; ClinicalTrials.gov Identifier: NCT03473223), which seeks to assess the efficacy of CSL112 on the risk of cardiovascular events. ${ }^{12,13}$ Prior trials have shown have shown CSL112 to be well-tolerated, ${ }^{14,15}$ and capable of increasing HDL cholesterol efflux in both healthy individuals and CAD patients. ${ }^{16-18}$ 
Human genetics can yield reliable evidence on the likely efficacy of pharmacological modification of a therapeutic target on risk of disease. For example, genetic variants in HMGCR and PCSK9 reliably elucidate the effects of statins and PCSK9 inhibition on circulating metabolic markers (including lipoproteins and their accompanying lipids) and risk of CAD. ${ }^{19-}$

${ }^{21}$ In this study, we used human genetics in five population-based cohorts with detailed lipoprotein profiling together with large-scale genome-wide data to identify a robust genetic instrument for apoA-I in order to provide reliable estimates of the potential causal effects of apoA-I on risk of CAD. We also compared our genetic results to observational associations of apoA-I and the risk of incident CAD in population-based cohorts and analyzed data from previously published RCTs of apoA-I infusion therapies reporting CAD events.

\section{METHODS}

\section{Datasets}

We used individual participant data including genotyping and lipoprotein profiling from five Finnish population-based cohorts, totaling up to 20,370 individuals (Supplemental Table 1): Northern Finland Birth Cohorts 22,231966 (NFBC66) and 1986 (NFBC86), the National FINRISK ${ }^{24}$ studies 1997 (FINRISK97) and 2007 (FINRISK07) and the Cardiovascular Risk in Young Finns Study ${ }^{25}$ (YFS). Details of the studies are provided in the Supplement. The genetic studies were conducted using data from these five Finnish cohorts. Observational estimates were assessed in the FINRISK cohorts (Supplemental Table 2). In addition, we used summarylevel data from large consortia ${ }^{26-29}$ as described in the Supplement. 


\section{Identifying genetic instruments for the circulating apoA-I concentration}

To find genetic variants that mimic the effect of apoA-I infusion therapies, we focused on cisacting protein quantitative trait loci (pQTLs) in the APOA1 locus, which contains the gene encoding apoA-I. We concentrated on the 1-Mb region flanking the APOA1 gene to find genetic variants associated with circulating apoA-I concentrations. Our initial approach was to count the number of single-nucleotide polymorphisms (SNPs) in this region and use this value to correct the multiple testing through a conventional Bonferroni approach, which corresponded to 7,210 SNPs and a Bonferroni-adjusted P-value of $<7 \times 10^{-6}$. However, the top SNP superseded this threshold and associated with apoA-I at conventional levels of GWAS significance $\left(P<5 \times 10^{-8}\right)$. To identify whether more than one cis-pQTL associated with apoA-I, we repeated the analysis of apoA-I concentrations on SNPs in/around APOA1 after conditioning on our top variant. We compared our results to those of a previous GWAS of apoA- $-^{30}$ and prioritized variant $\mathrm{rs} 12225230$ as the genetic instrument for apoA-I; this SNP was reported to be associated with apoA-I in the previous GWAS ${ }^{30}$ and summary statistics were available from this study. No additional conditionally-independent cis-pQTLs were identified. Details of the analyses and selection criteria are described in the Supplement.

\section{Assessment of pleiotropy of the genetic instrument}

To comprehensively annotate the metabolic effects of the genetic instrument for apoA-I, we investigated the effects of the genetic variant across a wide range of lipoprotein lipids and metabolites analyzed by nuclear magnetic resonance (NMR) spectroscopy (228 measures, Supplemental Table 4). ${ }^{31,32}$ The metabolites were first inverse-rank normalized, then adjusted for age, sex, and ten first principal components, and inverse rank-based normal transformation was used to transform the resulting residuals to a normal distribution to 
follow the normalization procedure of our previous GWAS. ${ }^{33}$ For the association of rs12225230 with metabolite levels, we considered $P$ values $<0.002$ as an evidence of association (Bonferroni correction with 22 principal components that explained $>95 \%$ of total variation in the levels of the metabolic measures). To facilitate comparison across multiple continuous measures, effect sizes are reported as standard deviation (SD) units per allele and illustrated as forest plots drawn with $\mathrm{R}, \mathrm{v}$. 3.4.3. To identify potential pleiotropic effects of the variant, we also conducted a phenome-wide association analysis in PhenoScanner. ${ }^{34}$

\section{Observational association of apoA-I with risk of incident coronary artery disease}

We estimated the association of serum apoA-I levels with risk of incident CAD (defined as MI, coronary revascularization, or death from CAD; see Supplement for details) in FINRISK97 ( $N=7,133$; number of incident CAD events 743 during 18-year follow-up) and FINRISK07 ( $N=4,402$; number of incident CAD events 175 during 8-year follow-up). ${ }^{24}$ To place the apoA-I association with CAD into context of other lipid measures, we also estimated the prospective associations of apolipoprotein B (apoB), HDL cholesterol and LDL cholesterol with CAD in the FINRISK97 cohort. Effect sizes were estimated using Cox proportional-hazard model and are reported in HR per SD difference in the circulating concentration. Two models were used: a minimally adjusted model with age and sex as covariates, and a fully adjusted model including a comprehensive set of covariates (age, sex, LDL cholesterol, body-mass index, systolic blood pressure, type 2 diabetes, smoking and alcohol consumption). The cohorts were meta-analyzed using inverse-variance fixed-effect modeling. All analyses were conducted using R, v. 3.4.3. 


\section{Analysis of randomized clinical trials of apoA-I infusion therapies}

To quantify the effect of apoA-I infusion from recent RCTs, we first identified trials in PubMed using the following search criterion: "apolipoprotein A-I infusion randomized clinical trial" (on 10/01/2018). We further assessed the articles identified in PubMed to identify placebo-controlled RCTs that reported major adverse cardiovascular events (MACEs) as endpoints (Supplemental Table 5). This identified the CHI-SQUARE ${ }^{35}$ trial $(3,6$, or 12 $\mathrm{mg} / \mathrm{kg}$ infusions of CER-001 weekly for six weeks) and the AEGIS-1 ${ }^{15}$ trial (2 or $6 \mathrm{~g}$ infusions of CSL112 weekly for four weeks). The analyzed end point was MACE defined as cardiac arrest, death, non-fatal MI, non-fatal stroke, coronary revascularization, or hospitalization for unstable angina or heart failure in the CHI-SQUARE trial; and as cardiovascular death, nonfatal MI, ischemic stroke or hospitalization for unstable angina in the AEGIS-I trial. The total number of MACE cases was 55 in CHI-SQUARE (total number of trial participants=486) and 74 in AEGIS-I (total $N=1,258$ ). We extracted estimates corresponding to all doses of apoA-I infusion therapies and calculated relative risks for MACEs for each dose separately.

\section{Assessing causality}

We assessed causality between the exposure (circulating apoA-I levels) and outcome (CAD) utilizing the Mendelian randomization (MR) framework (Supplemental Figure 1). ${ }^{36,37}$ We used a two-sample MR approach where summary data of the exposure (i.e., SNP to apoA-I level) and outcome (i.e., SNP to CAD) originate from different sources. ${ }^{36,37}$ Rs 12225230 located in the APOA1 locus was used as the genetic instrument. This approach included the use of the following estimates: [1] per-allele effect estimate (in $\mathrm{g} / \mathrm{L}$ ) for the association between rs12225230 and apoA-I (meta-analyzed result between the Finnish individual participant data (IPD) and a prior $\mathrm{GWAS}^{30}$; total $\mathrm{N}=37,093$ ); [2] per-allele effect estimates 
with risk of CAD from a large GWAS, i.e., meta-analysis of the UK Biobank with CARDIoGRAMplusC4D ${ }^{29}$ (case $\mathrm{N}=122,733$ ). To obtain a causal estimate and the corresponding standard error for the association between apoA-I and CAD, we used the formula described by Burgess et $a^{\beta 8}$ that collapses to Wald ratio for a single variant. To facilitate comparison with observational estimates, the causal estimate of apoA-I on risk of CAD was scaled to SD units, using SD of $0.225 \mathrm{~g} / \mathrm{L}$ (the SD of apoA-I in our largest cohort, FINRISK97) and assessed evidence of heterogeneity between the causal and observational estimates using Cochrane's Q statistic. In sensitivity analyses, we repeated the MR analysis using the per-allele effect estimate between rs12225230 and apoA-I using values derived from just the Finnish population cohorts and just the prior GWAS. ${ }^{30}$ We report our power to detect various effect estimates of apoA-I and risk of CAD in the MR analyses in Supplemental Table 3.

\section{RESULTS}

\section{Identification of genetic variants associated with circulating apoA-I concentrations}

Multiple SNPs in/around the APOA1 locus were associated with apoA-I serum concentration

(Figure 1, Supplemental Table 6) with the association peak spanning a 466-kb region. We identified the missense variant rs 12225230 to associate with apoA-I concentrations at GWAS significance (per-allele beta $0.076 \mathrm{SD}, \mathrm{P}=1.5 \times 10^{-9}$ ), which was in linkage disequilibrium (LD) with the top SNP rs625145 $\left(D^{\prime}=1.00, r^{2}=0.91\right)$. This missense variant was previously shown to be associated with apoA-I serum concentration ${ }^{30}$ and is mapped to the SIK3 gene encoding SIK family kinase 3 , located $20 \mathrm{~kb}$ upstream of APOA1. When we conditioned on rs 12225230 , there were no additional associations (Supplemental Figure 2), indicating that rs 12225230 or 
SNPs in LD with this SNP are likely the principal drivers of the apoA-I association in this locus. We estimated the effect of the minor allele of rs12225230 on apoA-I levels to be $0.0158 \mathrm{~g} / \mathrm{L}$ (SE 0.0030). In meta-analysis of our cohorts with a previous GWAS of apoA-I (reporting $0.0320 \mathrm{~g} / \mathrm{L}$ [SE 0.0033] increment per minor allele of rs12225230) ${ }^{30}$ the effect of the minor allele $\mathrm{C}$ of rs12225230 was estimated to be $0.0230 \mathrm{~g} / \mathrm{L}\left(\mathrm{SE} 0.0022 ; \mathrm{P}=1.5 \times 10^{-25}\right.$ ), corresponding to an F-statistic of 109.

When we further annotated this SNP, rs12225230 mapped to a region containing regulatory chromatin states and enhancer histone marks in several tissues, including the liver. In large eQTL data, there were no significant eQTLs in the APOA1 locus in the liver or small intestine. ${ }^{26,39}$ According to mQTL data ${ }^{40}, \mathrm{rs} 12225230$ is associated with the methylation status of nearby CpG methylation sites. Furthermore, rs12225230 is in LD with rs625145 (top SNP in our apoA-I GWAS), and methylation status of CpG sites associated with this SNP was previously reported to be causally associated with apoA-I concentrations. ${ }^{41}$

We assessed whether rs12225230 represents an unbiased instrument to mimic the effect of apoA-I increasing therapies by characterizing the effects of rs 12225230 on metabolic measures (Figure 2, Supplemental Figure 3). We detected that, in addition to associating with higher apoA-I, the minor allele of rs12225230 was associated with higher levels of multiple HDL-related measures, including, e.g., concentration of large, medium and small HDL particles (L-HDL-P, beta 0.055 SD, P=1.2E-05; M-HDL-P, beta 0.061 SD, P=1.2E-06; SHDL-P, beta 0.055 SD, $\mathrm{P}=1.2 \mathrm{E}-05$ ), and HDL cholesterol (beta $0.070 \mathrm{SD}, \mathrm{P}=2.5 \mathrm{E}-08$ ). In addition, rs12225230 was associated, e.g., with fatty acid measurements (e.g., polyunsaturated fatty acids, beta $0.048 \mathrm{SD}, \mathrm{P}=1.2 \mathrm{E}-04)$; associations with the fatty acids 
likely reflect their associations with multiple HDL-related traits. In contrast, rs12225230 was not associated with the majority of apoB related traits (Supplemental Figure 3).

We further investigated whether the association of rs12225230 with apoA-l level could be confounded by associations with other phenotypes. To this end, we screened publicly available genotype-phenotype associations using PhenoScanner. ${ }^{34}$ The associations of rs12225230 were not likely confounded by conventional risk factors (see Supplemental Methods, Supplemental Table 7, Supplemental Figure 4). ${ }^{42,43}$

\section{Observational associations of apoA-I levels with incident coronary artery disease}

We estimated the prospective observational associations of apoA-I with risk of CAD in the FINRISK97 cohort ( $\mathrm{N}=7,133$, with 743 incident CAD events), and compared the association of apoA-I to the corresponding CAD associations for apoB, HDL and LDL cholesterol. ApoA-I (HR 0.86 per 1-SD higher apoA-I; 95\%Cl 0.79-0.93, Figure 3) had a similar magnitude of association with CAD as did HDL cholesterol (HR 0.79 per 1-SD higher HDL-C; 95\% Cl 0.720.86), apoB (HR 0.84 per 1-SD lower apoB; 95\%Cl 0.79-0.91) and LDL cholesterol (HR 0.88 per 1-SD lower LDL-C; 95\%Cl 0.82-0.95). The prospective association of apoA-I with risk of CAD was similar in minimally (age and sex as covariates) and fully (age, sex, body mass index, systolic blood pressure, type 2 diabetes, smoking and alcohol consumption as covariates) adjusted models (Supplemental Table 8). ApoA-I showed a dose-response relationship, with the risk of CAD being HR $0.57(95 \% \mathrm{Cl} 0.45-0.75)$ comparing the highest to lowest quintiles of apoA-I (Figure 3B). 
In meta-analysis of the FINRISK97 and FINRISK07 cohorts (total 918 incident CAD cases), higher concentrations of apoA-I associated with a lower risk of CAD (HR 0.81 per 1-SD higher apoA-I, 95\% $\mathrm{Cl}$ 0.75-0.88) after adjustment for age, sex, LDL cholesterol, body-mass index, systolic blood pressure, type 2 diabetes, smoking and alcohol consumption. The associations were similar in minimally and fully adjusted models and in both cohorts (Supplemental Figure 5). ApoA-I showed only low correlation ( $r$ between -0.3 and 0.3 ) with the potential confounders included as covariates in the models (Supplemental Figure 6).

\section{Effects of apoA-I infusion therapies on major adverse cardiovascular events}

We appraised the evidence base on the relationship of apoA-I infusion therapies and risk of cardiovascular disease in two phase II RCTs reporting major adverse cardiovascular event (MACE) endpoints: the CHI-SQUARE ${ }^{35}$ and AEGIS-I ${ }^{15}$ trials. None of the apoA-I infusion doses reduced the risk of MACEs: while dose-specific effect estimates had wide $95 \%$ confidence intervals, it is noteworthy that at all doses investigated, the point estimates were on the opposite side of the null that would indicate a potential protective effect (Supplemental Table 9).

\section{Evaluation of the causal role of serum apoA-I in coronary artery disease}

Using data from a large GWAS of coronary artery disease including 122,733 CAD cases in 547,261 individuals (meta-analysis of UK Biobank CAD GWAS with CARDIoGRAMplusC4D29), rs1222530 was not associated with risk of CAD (per-allele log-odds 0.0124, SE 0.0073, $P=0.090)$. Similarly, no significant association of $r s 1222530$ was reported in any of the large GWAS for cardiovascular phenotypes, including CAD, MI or stroke (Supplemental Table 10). 
Using the rs1222530-apoA-I and rs1222530-CAD associations, we did not find evidence to support a causal role of apoA-I with risk of CAD: the causal estimate (calculated based on meta-analysis of our own GWAS and a previous GWAS of apoA-1 $1^{30}$ ) for CAD was an OR of 1.13 per 1-SD higher apoA-I level, 95\%Cl 0.98-1.30 (Figure 4, Supplemental Figure 7) which differed (P-heterogeneity<0.001) to the corresponding fully-adjusted observational estimate (HR 0.81; 95\%Cl: 0.75-0.88). Causal estimates for CAD were similar when calculated based on the apoA-I effect estimates from the previous $\mathrm{GWAS}^{30}$ (OR 1.09, 95\% Cl 0.99-1.21) and our own GWAS (OR 1.19, 95\%CI 0.97-1.46).

\section{DISCUSSION}

Our findings do not provide genetic support for the hypothesis that circulating apoA-I concentrations play a protective role in the etiology of CAD. Together with previous genetic and randomized controlled trial evidence indicating that HDL cholesterol does not have a protective effect on risk of $C A D, 1,2,5,6$ these results suggest that apoA-I is unlikely to represent a valid therapeutic approach for the prevention and treatment of CAD. The implication of these findings is that the ongoing AEGIS-II phase III cardiovascular outcome trial of CSL112 is unlikely to show evidence of vascular benefit.

HDL cholesterol and apoA-I are among the serum lipoprotein measures showing strongest inverse associations with cardiovascular disease in epidemiological studies. ${ }^{44,45}$ However, large phase III cardiovascular outcome RCTs using HDL cholesterol increasing therapies have failed to identify that the risk of CAD is proportionate to the amount by which HDL cholesterol is increased, ${ }^{1,2}$ and, consistently, studies of human genetics find that the 
association of HDL cholesterol with CAD is not causal. ${ }^{5,6}$ In the REVEAL trial of the cholesteryl ester transfer protein (CETP) inhibitor, anacetrapib, although treatment with anacetrapib did increase HDL cholesterol, the cardiovascular benefit was proportionate to the degree of apoB lowering, rather than to the effect on HDL cholesterol. ${ }^{3}$ Here, we add further evidence showing that targeting HDL-related pathways through increases in apoA-I is unlikely to be effective in lowering risk of CAD. We show that long-term genetically increased circulating apoA-I levels do not protect from CAD, a finding consistent with the cardiovascular associations in two trials of apoA-I infusion products (MDCO-216 and CER-001) that led to the termination of development of those products. ${ }^{10,11}$ Thus, randomized evidence of both the short-term, transient increase in circulating concentration of apoA-I arising from apoA-I infusions and the life-long increase caused by genetic variants fails to provide evidence in support of a protective role of apoA-I in CAD.

This study has several strengths. The SNP used to mimic the effect of apoA-I-increasing therapy, rs12225230, was identified in a locus-specific analysis of SNPs in/around APOA1 in meta-analysis of five Finnish cohorts in our analyses and the same variant was identified in a prior GWAS of apoA-1 ${ }^{30}$ with the mechanism thought to be related to alterations in methylation of regulatory sites. Having an F-statistic of 100, and identified through scanning genetic variants in the cis-encoding locus, the SNP represents a robust and valid genetic instrument for apoA-I. By only considering cis-acting pQTL SNPs from the APOA1 locus, we minimized the potential for confounding by trans-acting variants, since the use of cis-acting pQTLs as instrumental variables for proteins represents among the most reliable of approaches to Mendelian randomization. ${ }^{37}$ In addition, we carefully assessed the effects of rs12225230 on a detailed panel of serum metabolic measures as a means of providing 
comprehensive evidence against potential confounding by circulating metabolic markers other than those related to HDL pathways, and supplemented this by examining potential associations using publically available genetic repositories. For all analyses, we utilized large, well-characterized Finnish population cohorts and the largest available international GWAS consortia data for CAD to maximize statistical power. Taken together, our statistical and genetic approach yielded reliable estimates of the causal effect of apoA-I on risk of CAD and facilitated the discovery that, unlike the observational association, which is vulnerable to confounding and bias, apoA-I is unlikely to play a causal role in CAD.

The main focus of investigation was to provide genetic evidence on the hypothesis of whether apoA-I infusions protect from CAD, and to do so, we developed a genetic instrument that strongly and specifically associates with circulating apoA-I concentrations as the primary exposure. However, the rationale behind apoA-I-infusion therapies is that increases in apoA-I ought to promote cholesterol efflux from arterial wall macrophages, a process that can be quantitatively measured by HDL cholesterol efflux capacity (HDL-CEC). Similar to HDL cholesterol and apoA-I, HDL-CEC is inversely associated with risk of CAD. 7,46 Whether or not HDL-CEC is modified by apoA-I in a cause and effect manner is not currently known. The AEGIS trial showed that apoA-I infusion therapy with CSL112 led to higher concentrations of apoA-I and higher HDL-CEC, ${ }^{15,18}$ which suggests that higher HDL-CEC may represent a target-mediated effect of elevations in apoA-I. If this were true, then we would expect that our genetic instrument for higher apoA-I would also lead to higher HDL-CEC. This therefore means that we would expect that any effect of apoA-I infusion therapy on HDLCEC ought to be represented by our genetic instrument. 
A common criticism of drug-target Mendelian randomization analyses is that the genetic association with the biomarker of interest (in this case apoA-I) is typically small, and thus meaningful deductions cannot be made about the likely impact of modifying apoA-I through a therapeutic (such as an infusion), where the effect of the intervention on the biomarker of interest is typically much larger. The counterargument is that atherosclerosis is a life-long disease, and exposure to a small but consistent genetic elevation in apoA-I over several decades ought to lead to a lower risk of vascular disease if apoA-I is truly cardioprotective. It is this 'cumulative' exposure in the setting of a disease with a long latency period (such as cardiovascular disease) which means that 'physiological' risk factors such as LDL cholesterol tend to have much larger effects on risk of CVD from Mendelian randomization than from conventional observational studies. ${ }^{37}$ In our study, we had very good power ( 80\%) to detect an effect estimate from Mendelian randomization which was similar to that which we identified from our observational analyses. If apoA-I were truly causal, we would expect the effect estimate representing the actual causal estimate to be of greater magnitude (e.g. odds ratios of 0.7 or lower) for which we had excellent power. Our genetic analyses might give rise to a false negative in the context of 2-sample MR using non-overlapping datasets if we had a weak instrument, however our F-statistic was over 100, making this unlikely. A false negative might also arise were there to be a physiological threshold above which apoAI is cardioprotective but below which it has no effect. To investigate the plausibility of such a threshold effect, our observational analyses, in which we generated quintiles based on measured values of apoA-I, showed a clear dose-response relationship between apoA-I and risk of vascular disease, which argues against such a threshold effect. 
This study provides an example of using human genetics to guide the development of therapeutic targets, and predict the effects of phase III cardiovascular outcome trials. We note the similarity to other 'negative' MR studies of secretory phospholipase IIA- $A_{2}{ }^{47}$ and Lp$\mathrm{PLA}_{2}{ }^{48}$, both of which were the focus of large-scale phase III cardiovascular outcome trials. In both cases, reliable genetic evidence might have prevented vast sums of money from being spent on phase III cardiovascular outcome trials of therapies modifying drug targets that were destined to fail due to lack of target-mediated efficacy. While the biological plausibility of the hypothesis that apoA-I infusion might lower risk of CAD through increased reverse cholesterol transport is biologically appealing, our genetic evidence indicates that the ongoing AEGIS-II phase III trial is unlikely to succeed in lowering the risk of CAD. The cumulative evidence therefore brings into question whether pharmaceutical research and development should continue to invest in developing apoA-I infusion therapies for the purposes of preventing CAD.

In conclusion, our findings do not support the hypothesis that increasing circulating apoA-I concentrations represents a valid approach for the prevention of CAD. These results add to the burgeoning evidence that refutes a protective role of HDL and HDL-related phenotypes in the etiology of CAD.

Sources of funding: MAK is supported by a Senior Research Fellowship from the National Health and Medical Research Council (NHMRC) of Australia (APP1158958). He also works in a unit that is supported by the University of Bristol and UK Medical Research Council (MC_UU_12013/1). The Baker Institute is supported in part by the Victorian Government's 
Operational Infrastructure Support Program. JK is supported through funds from the Academy of Finland (grant numbers 297338 and 307247) and the Novo Nordisk Foundation (grant number NNF170C0026062). MVH works in a unit that receives funding from the UK Medical Research Council and is supported by a British Heart Foundation Intermediate Clinical Research Fellowship (FS/18/23/33512) and the National Institute for Health Research Oxford Biomedical Research Centre. QW is supported by a Postdoctoral Fellowship from the Novo Nordisk Foundation (NNF170C0027034). VS is supported by the Finnish Foundation for Cardiovascular Research. KK is supported by the Academy of Finland (grant number 250207). The Young Finns Study has been financially supported by the Academy of Finland: grants 286284, 134309 (Eye), 126925, 121584, 124282, 129378 (Salve), 117787 (Gendi), and 41071 (Skidi); the Social Insurance Institution of Finland; Competitive State Research Financing of the Expert Responsibility area of Kuopio, Tampere and Turku University Hospitals (grant X51001); Juho Vainio Foundation; Paavo Nurmi Foundation; Finnish Foundation for Cardiovascular Research; Finnish Cultural Foundation; The Sigrid Juselius Foundation; Tampere Tuberculosis Foundation; Emil Aaltonen Foundation; Yrjö Jahnsson Foundation; Signe and Ane Gyllenberg Foundation; Diabetes Research Foundation of Finnish Diabetes Association; EU Horizon 2020 (grant 755320 for TAXINOMISIS); European Research Council (grant 742927 for MULTIEPIGEN project); and Tampere University Hospital Supporting Foundation. NFBC66 received financial support from University of Oulu Grant no. 65354; Oulu University Hospital Grant no. 2/97, 8/97; Ministry of Health and Social Affairs Grant no. 23/251/97, 160/97, 190/97; National Institute for Health and Welfare, Helsinki Grant no. 54121; and Regional Institute of Occupational Health, Oulu, Finland Grant no. 50621, 54231. NFBC86 was supported by the following grants: EU QLG1-CT-2000-01643 (EUROBLCS) Grant no. E51560; NorFA Grants no. 731, 20056, 30167; and USA / NIHH 2000 G DF682 Grant no. 
50945. No funders or sponsors were involved in the design and conduct of the study; collection, management, analysis, and interpretation of the data; preparation, review, or approval of the manuscript; or decision to submit the manuscript for publication.

Disclosures: VS has participated in a conference trip sponsored by Novo Nordisk and received an honorarium for participating in an advisory board meeting (unrelated to the present study). He also has ongoing research collaboration with Bayer Ltd (unrelated to the present study). No other authors reported disclosures. 


\section{References}

1. Schwartz GG, Olsson AG, Abt M, et al. Effects of dalcetrapib in patients with a recent acute coronary syndrome. N Engl J Med. 2012;367:2089-2099.

2. HPS2-THRIVE Collaborative Group, Landray MJ, Haynes R, Hopewell JC, Parish S, Aung T, Tomson J, Wallendszus K, Craig M, Jiang L, Collins R, Armitage J. Effects of extended-release niacin with laropiprant in high-risk patients. N Engl J Med. 2014;371:203-212.

3. HPS3/TIMI55-REVEAL Collaborative Group, Bowman L, Hopewell JC, Chen F, Wallendszus

K, Stevens W, Collins R, Wiviott SD, Cannon CP, Braunwald E, Sammons E, Landray MJ.

Effects of anacetrapib in patients with atherosclerotic vascular disease. $N$ Engl J Med. 2017;377:1217-1227.

4. Lincoff AM, Nicholls SJ, Riesmeyer JS, et al. Evacetrapib and cardiovascular outcomes in high-risk vascular disease. N Engl J Med. 2017;376:1933-1942.

5. Holmes MV, Asselbergs FW, Palmer TM, et al. Mendelian randomization of blood lipids for coronary heart disease. Eur Heart J. 2015;36:539-550.

6. Voight BF, Peloso GM, Orho-Melander M, et al. Plasma HDL cholesterol and risk of myocardial infarction: A mendelian randomisation study. Lancet. 2012;380:572-580.

7. Rohatgi A, Khera A, Berry JD, Givens EG, Ayers CR, Wedin KE, Neeland IJ, Yuhanna IS, Rader DR, de Lemos JA, Shaul PW. HDL cholesterol efflux capacity and incident cardiovascular events. N Engl J Med. 2014;371:2383-2393.

8. Gillard BK, Rosales C, Xu B, Gotto AM, Pownall HJ. Rethinking reverse cholesterol transport and dysfunctional high-density lipoproteins. J Clin Lipidol. 2018;12:849-856. 
9. Winslow R. Pfizer Agrees to Acquire Esperion for \$1.3 Billion. Wall Street Journal.

Published December 22, 2003. https://www.wsj.com/articles/SB107203106453468800.

10. Nicholls SJ, Andrews J, Kastelein JJP, et al. Effect of serial infusions of CER-001, a pre-beta high-density lipoprotein mimetic, on coronary atherosclerosis in patients following acute coronary syndromes in the CER-001 atherosclerosis regression acute coronary syndrome trial: A randomized clinical trial. JAMA Cardiol. 2018;3:815-822.

11. Nicholls SJ, Puri R, Ballantyne CM, Jukema JW, Kastelein JJP, Koenig W, Wright RS, Kallend D, Wijngaard P, Borgman M, Wolski K, Nissen SE. Effect of infusion of high-density lipoprotein mimetic containing recombinant apolipoprotein A-I milano on coronary disease in patients with an acute coronary syndrome in the MILANO-PILOT trial: A randomized clinical trial. JAMA Cardiol. 2018;3:806-814.

12. Diditchenko S, Gille A, Pragst I, Stadler D, Waelchli M, Hamilton R, Leis A, Wright SD. Novel formulation of a reconstituted high-density lipoprotein (CSL112) dramatically enhances ABCA1-dependent cholesterol efflux. Arterioscler Thromb Vasc Biol. 2013;33:22022211.

13. Gibson CM, Korjian S, Tricoci P, Daaboul Y, Alexander JH, Steg PG, Lincoff AM, Kastelein JJ, Mehran R, D'Andrea D, Merkely B, Zarebinski M, Ophius TO, Harrington RA. Rationale and design of apo-I event reduction in ischemic syndromes I (AEGIS-I): A phase 2b, randomized, placebo-controlled, dose-ranging trial to investigate the safety and tolerability of CSL112, a reconstituted, infusible, human apoA-I, after acute myocardial infarction. Am Heart J. $2016 ; 180: 22-28$ 
14. Easton R, Gille A, D'Andrea D, Davis R, Wright SD, Shear C. A multiple ascending dose study of CSL112, an infused formulation of ApoA-I. J Clin Pharmacol. 2014;54:301-310.

15. Michael Gibson C, Korjian S, Tricoci P, et al. Safety and tolerability of CSL112, a reconstituted, infusible, plasma-derived apolipoprotein A-I, after acute myocardial infarction: The AEGIS-I trial (ApoA-I event reducing in ischemic syndromes I). Circulation. 2016;134:1918-1930.

16. Gille A, Easton R, D'Andrea D, Wright SD, Shear CL. CSL112 enhances biomarkers of reverse cholesterol transport after single and multiple infusions in healthy subjects. Arterioscler Thromb Vasc Biol. 2014;34:2106-2114.

17. Didichenko SA, Navdaev AV, Cukier AM, Gille A, Schuetz P, Spycher MO, Therond P, Chapman MJ, Kontush A, Wright SD. Enhanced HDL functionality in small HDL species produced upon remodeling of HDL by reconstituted HDL, CSL112: Effects on cholesterol efflux, anti-inflammatory and antioxidative activity. Circ Res. 2016;119:751-763.

18. Gille A, D'Andrea D, Tortorici MA, Hartel G, Wright SD. CSL112 (apolipoprotein A-I [human]) enhances cholesterol efflux similarly in healthy individuals and stable atherosclerotic disease patients. Arterioscler Thromb Vasc Biol. 2018;38:953-963.

19. Voora D, Shah SH. Pharmacometabolomics meets genetics: A "natural" clinical trial of statin effects. J Am Coll Cardiol. 2016;67:1211-1213.

20. Wurtz $P$, Wang $Q$, Soininen $P$, et al. Metabolomic profiling of statin use and genetic inhibition of HMG-CoA reductase. J Am Coll Cardiol. 2016;67:1200-1210. 
21. Sliz E, Kettunen J, Holmes MV, et al. Metabolomic consequences of genetic inhibition of PCSK9 compared with statin treatment. Circulation. 2018;138:2499-2512.

22. Jarvelin MR, Sovio U, King V, Lauren L, Xu B, McCarthy MI, Hartikainen AL, Laitinen J, Zitting P, Rantakallio P, Elliott P. Early life factors and blood pressure at age 31 years in the 1966 northern finland birth cohort. Hypertension. 2004;44:838-846.

23. Jarvelin MR, Hartikainen-Sorri AL, Rantakallio P. Labour induction policy in hospitals of different levels of specialisation. Br J Obstet Gynaecol. 1993;100:310-315.

24. Borodulin $\mathrm{K}$, Vartiainen $\mathrm{E}$, Peltonen $\mathrm{M}$, Jousilahti $\mathrm{P}$, Juolevi A, Laatikainen $\mathrm{T}$, Mannisto $\mathrm{S}$, Salomaa V, Sundvall J, Puska P. Forty-year trends in cardiovascular risk factors in finland. Eur J Public Health. 2015;25:539-546.

25. Raitakari OT, Juonala M, Ronnemaa T, et al. Cohort profile: The cardiovascular risk in young finns study. Int J Epidemiol. 2008;37:1220-1226.

26. GTEx Consortium. The genotype-tissue expression (GTEx) project. Nat Genet. 2013;45:580-585.

27. Nelson CP, Goel A, Butterworth AS, et al. Association analyses based on false discovery rate implicate new loci for coronary artery disease. Nat Genet. 2017;49:1385-1391.

28. Malik R, Chauhan G, Traylor M, et al. Multiancestry genome-wide association study of 520,000 subjects identifies 32 loci associated with stroke and stroke subtypes. Nat Genet. 2018;50:524-537. 
29. van der Harst $P$, Verweij N. Identification of 64 novel genetic loci provides an expanded view on the genetic architecture of coronary artery disease. Circ Res. 2018;122:433-443.

30. Chasman DI, Pare G, Mora S, Hopewell JC, Peloso G, Clarke R, Cupples LA, Hamsten A, Kathiresan S, Malarstig A, Ordovas JM, Ripatti S, Parker AN, Miletich JP, Ridker PM. Fortythree loci associated with plasma lipoprotein size, concentration, and cholesterol content in genome-wide analysis. PLoS Genet. 2009;5:e1000730.

31. Soininen P, Kangas AJ, Wurtz P, Suna T, Ala-Korpela M. Quantitative serum nuclear magnetic resonance metabolomics in cardiovascular epidemiology and genetics. Circ Cardiovasc Genet. 2015;8:192-206.

32. Wurtz P, Kangas AJ, Soininen P, Lawlor DA, Davey Smith G, Ala-Korpela M. Quantitative serum nuclear magnetic resonance metabolomics in large-scale epidemiology: A primer on omic technologies. Am J Epidemiol. 2017;186:1084-1096.

33. Kettunen J, Demirkan A, Wurtz P, et al. Genome-wide study for circulating metabolites identifies 62 loci and reveals novel systemic effects of LPA. Nat Commun. 2016;7:11122.

34. Staley JR, Blackshaw J, Kamat MA, Ellis S, Surendran P, Sun BB, Paul DS, Freitag D, Burgess S, Danesh J, Young R, Butterworth AS. PhenoScanner: A database of human genotype-phenotype associations. Bioinformatics. 2016;32:3207-3209.

35. Tardif JC, Ballantyne CM, Barter P, et al. Effects of the high-density lipoprotein mimetic agent CER-001 on coronary atherosclerosis in patients with acute coronary syndromes: A randomized trial. Eur Heart J. 2014;35:3277-3286. 
36. Davey Smith G, Hemani G. Mendelian randomization: Genetic anchors for causal inference in epidemiological studies. Hum Mol Genet. 2014;23:89.

37. Holmes MV, Ala-Korpela M, Smith GD. Mendelian randomization in cardiometabolic disease: Challenges in evaluating causality. Nat Rev Cardiol. 2017;14:577-590.

38. Burgess S, Butterworth A, Thompson SG. Mendelian randomization analysis with multiple genetic variants using summarized data. Genet Epidemiol. 2013;37:658-665.

\section{Strunz T, Grassmann F, Gayan J, Nahkuri S, Souza-Costa D, Maugeais C, Fauser S,} Nogoceke E, Weber BHF. A mega-analysis of expression quantitative trait loci (eQTL) provides insight into the regulatory architecture of gene expression variation in liver. Sci Rep. 2018;8:z.

40. Gaunt TR, Shihab HA, Hemani G, Min JL, Woodward G, Lyttleton O, Zheng J, Duggirala A, McArdle WL, Ho K, Ring SM, Evans DM, Davey Smith G, Relton CL. Systematic identification of genetic influences on methylation across the human life course. Genome Biol. 2016;17:z.

41. Richardson TG, Zheng J, Davey Smith G, Timpson NJ, Gaunt TR, Relton CL, Hemani G. Mendelian randomization analysis identifies $\mathrm{CpG}$ sites as putative mediators for genetic influences on cardiovascular disease risk. Am J Hum Genet. 2017;101:590-602.

42. Scott RA, Scott LJ, Magi R, et al. An expanded genome-wide association study of type 2 diabetes in europeans. Diabetes. 2017;66:2888-2902.

43. http://www.nealelab.is/uk-biobank/. 
44. Wurtz $P$, Havulinna AS, Soininen $P$, et al. Metabolite profiling and cardiovascular event risk: A prospective study of 3 population-based cohorts. Circulation. 2015;131:774-785.

45. Holmes MV, Millwood IY, Kartsonaki C, et al. Lipids, lipoproteins, and metabolites and risk of myocardial infarction and stroke. J Am Coll Cardiol. 2018;71:620-632.

46. Khera AV, Demler OV, Adelman SJ, Collins HL, Glynn RJ, Ridker PM, Rader DJ, Mora S. Cholesterol efflux capacity, high-density lipoprotein particle number, and incident cardiovascular events: An analysis from the JUPITER trial (justification for the use of statins in prevention: An intervention trial evaluating rosuvastatin). Circulation. 2017;135:24942504.

47. Holmes MV, Simon T, Exeter HJ, et al. Secretory phospholipase A(2)-IIA and cardiovascular disease: A mendelian randomization study. J Am Coll Cardiol. 2013;62:19661976.

48. Millwood IY, Bennett DA, Walters RG, et al. Lipoprotein-associated phospholipase A2 loss-of-function variant and risk of vascular diseases in 90,000 chinese adults. J Am Coll Cardiol. 2016;67:230-231. 


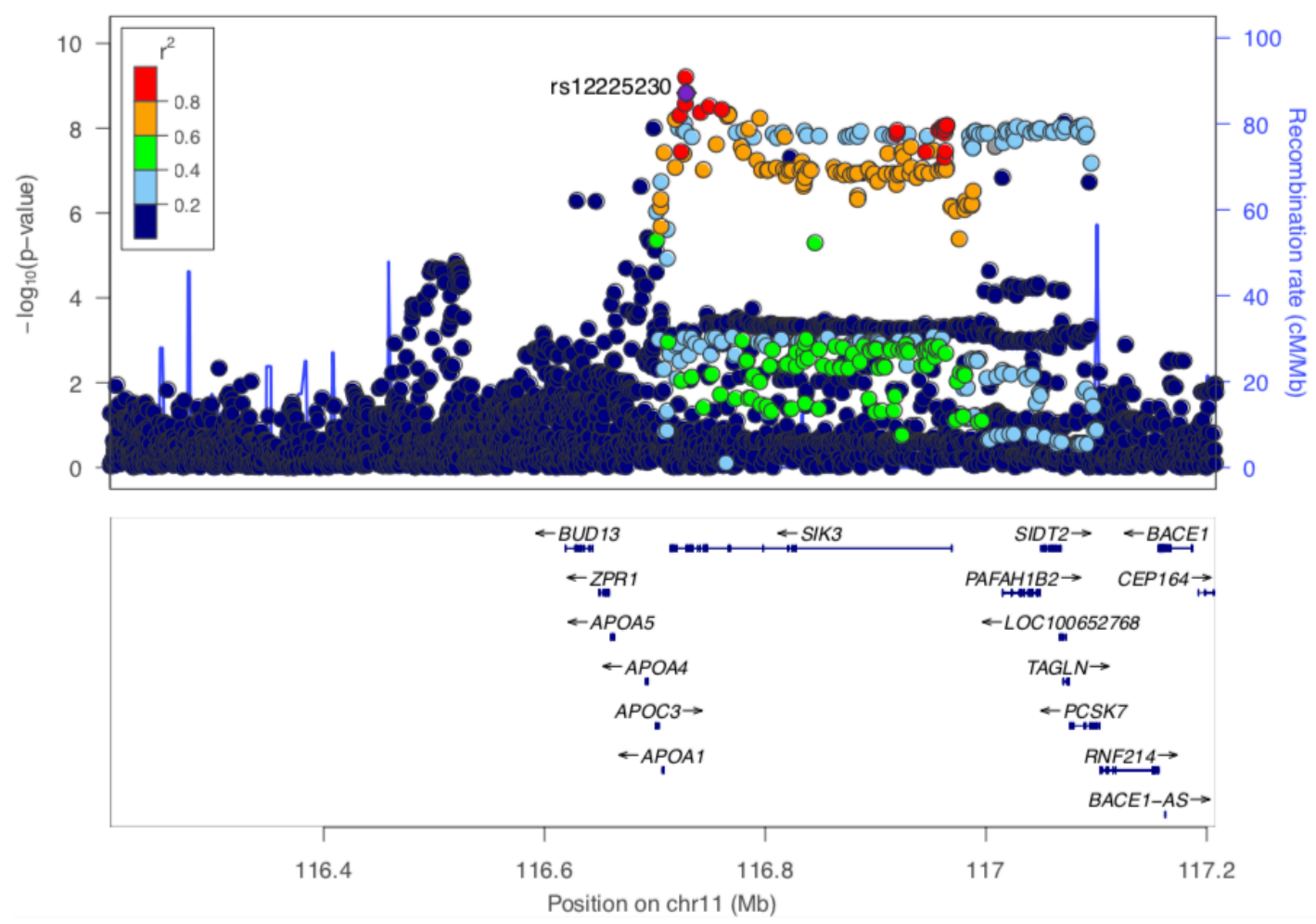

Figure 1. Association of SNPs in the APOA1 locus with circulating concentrations of apoA-I.

Each dot represents the association of a single genetic variant with apoA-I levels in metaanalysis of five Finnish population cohorts $(-\log 10$ of $P$ value shown on the $y$ axis and chromosomal position on the $\mathrm{x}$ axis). The $500-\mathrm{kb}$ region flanking APOA1 is shown.

Rs12225230, highlighted in violet and was used as an instrument in this study, is robustly associated with apoA-I $\left(P=2 \times 10^{-9}\right)$ and is in LD with the top variant $r 5625145\left(P=6 \times 10^{-10}\right)$. 

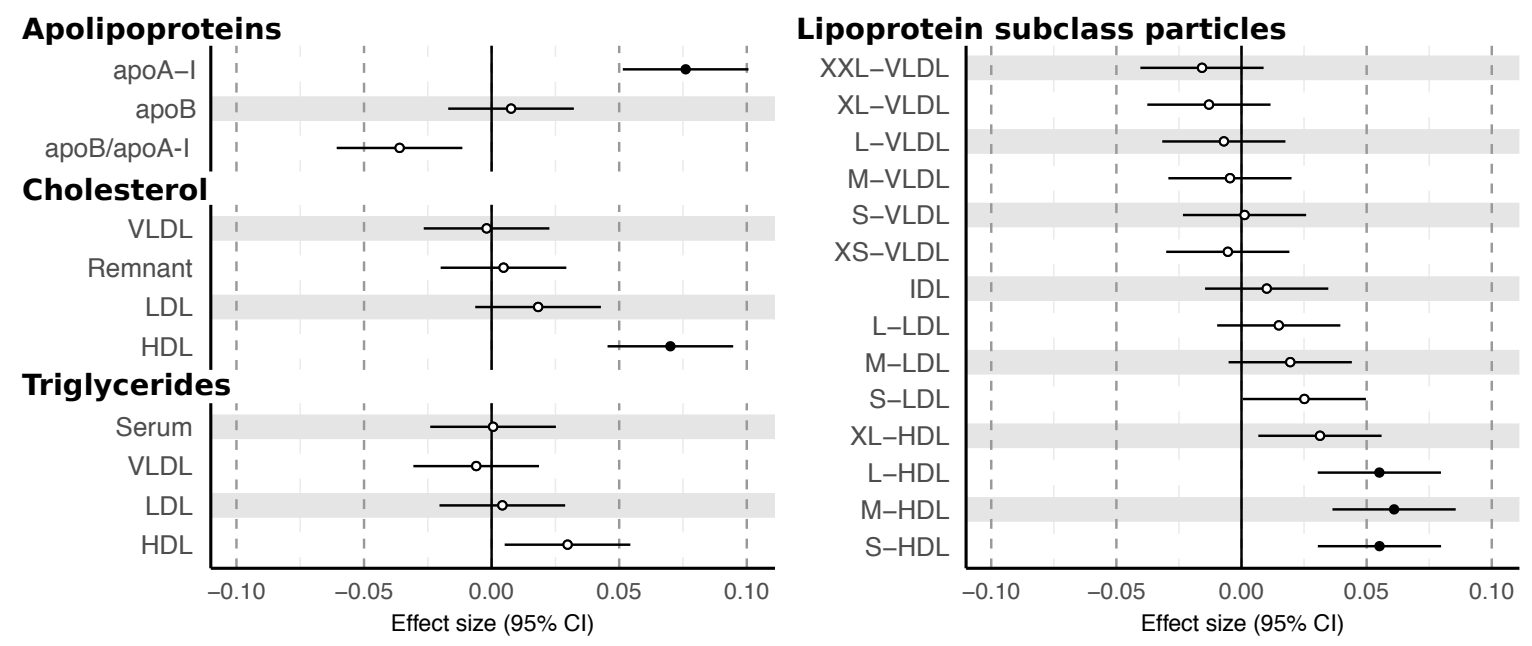

Figure 2. Association of rs12225230 with key lipoprotein-related concentration measures.

Remnant cholesterol refers to cholesterol carried in VLDL and IDL particles. Effect sizes are estimated as SD differences in metabolite concentrations per rs12225230-C allele. Closed symbols, $\mathrm{P}<0.002$ (significant association); open symbols $\mathrm{P} \geq 0.002$. Abbreviations: apoA-I, apolipoprotein A-I; apoB, apolipoprotein B; VLDL, very low-density lipoprotein, LDL, lowdensity lipoprotein; HDL, high-density lipoprotein; IDL, intermediate-density lipoprotein. 
(A) Comparison to key lipoprotein measures

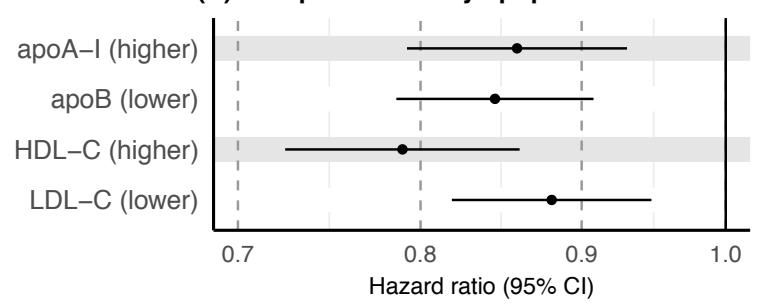

(B) Dose response relationship of apoA-I

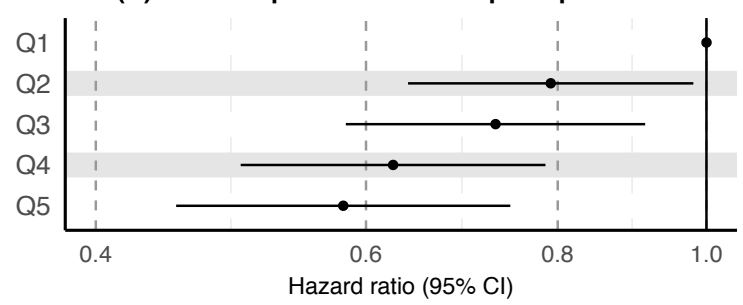

Figure 3. Observational associations of apoA-I and risk of coronary artery disease. Panel A: the association of apoA-I with CAD risk was compared to those of key lipoprotein measures (apoB, HDL and LDL cholesterol). Note, the effects of apoB and LDL cholesterol are inversed; i.e., the effects are shown per 1-SD reduction in apoB and LDL cholesterol. Associations were assessed with 743 cases and 6,390 controls in FINRISK97, with adjustment for age, sex, bodymass index, systolic blood pressure, type 2 diabetes, smoking and alcohol consumption. Associations are presented as HR $(95 \% \mathrm{Cl})$ per 1-SD lipoprotein measure. Panel B: Doseresponse association of apoA-I with risk of CAD. Associations were additionally adjusted for LDL cholesterol. Effect estimates are presented per quintile of apoA-I with the lowest quintile (Q1) used as the reference category. 


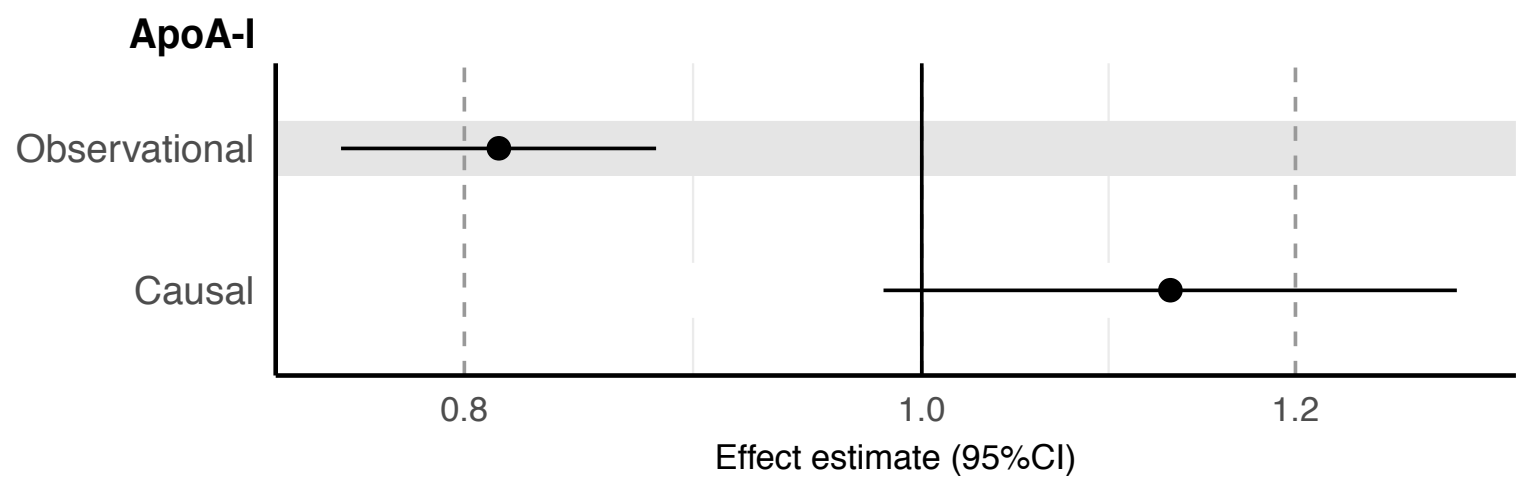

Figure 4. Observational and causal effects of circulating apoA-I levels and risk of coronary artery disease. Observational estimate was assessed in the FINRISK97 and FINRISK07 cohorts (918 coronary artery disease cases among 15,353 individuals) with age, sex, LDL cholesterol, body-mass index, systolic blood pressure, type 2 diabetes, smoking and alcohol consumption as covariates. Causal estimate was calculated based on association of rs12225230 with apoA-I levels (effect estimate from meta-analysis of five Finnish cohorts and a previous $\mathrm{GWAS}^{30}$, total $\mathrm{N}=37,493$ ) and coronary artery disease (effect estimate from meta-analysis of the UK Biobank and CARDIoGRAMplusC4D ${ }^{29} ; 122,733$ CAD cases among 547,261 individuals). Effect estimates correspond to the risk (observational association: hazard ratio, HR; causal estimate: odds ratio, OR) of CAD per 1-SD higher apoA-I (equivalent to $0.225 \mathrm{~g} / \mathrm{L}$ apoA-I). 